Very low power, high voltage base for a Photo Multiplier Tube for the KM3NeT deep sea neutrino telescope

This article has been downloaded from IOPscience. Please scroll down to see the full text article. 2010 JINST 5 C12049

(http://iopscience.iop.org/1748-0221/5/12/C12049)

View the table of contents for this issue, or go to the journal homepage for more

Download details:

IP Address: 137.138.124.142

The article was downloaded on 11/07/2011 at 12:41

Please note that terms and conditions apply. 


\title{
Very low power, high voltage base for a Photo Multiplier Tube for the KM3NeT deep sea neutrino telescope
}

\author{
P. Timmer, ${ }^{1}$ E. Heine and H. Peek \\ Nikhef, \\ Science Park 105, 1098 XG Amsterdam, The Netherlands \\ E-mail: pault@nikhef.nl
}

ABSTRACT: The described system is developed in the framework of a deep-sea submerged Very Large Volume neutrino Telescope where photons are detected by a large number of Photo Multiplier Tubes. These PMTs are placed in optical modules (OM). A basic Cockcroft-Walton (CW) voltage multiplier circuit design is used to generate multiple voltages to drive the dynodes of the photomultiplier tube. To achieve a long lifetime and a high reliability the dissipation in the OM must be kept to the minimum. The design is also constrained by size restrictions, load current, voltage range, and the maximum allowable ripple in the output voltage. A surface mount PMT-base PCB prototype is designed and successfully tested. The system draws less than $1.5 \mathrm{~mA}$ of supply current at a voltage of $3.3 \mathrm{~V}$ with outputs up to $-1400 \mathrm{Vdc}$ cathode voltage, a factor 10 less than the commercially available state of the art.

KEYWORDS: Photon detectors for UV, visible and IR photons (vacuum); Photon detectors for UV, visible and IR photons (vacuum) (photomultipliers, HPDs, others)

\footnotetext{
${ }^{1}$ Corresponding author.
} 


\section{Contents}

1 Introduction 1

2 System components 2

2.1 Photo Multiplier Tube 2

2.2 Cockcroft Walton multiplier (CW) 3

2.3 Flyback converter 3

3 Design 3

4 Results 4

4.1 Waveforms 4

5 Outlook 5

\section{Introduction}

The purpose of the PMT is conversion of photons from Cherenkov radiation. The Cherenkov light emitted by secondary charged particles (muons) created via a neutrino interaction in or near an optically transparent medium, such as water or ice is used to detect neutrinos. Cherenkov light is detected at the single-photon level, where a precise measurement of place and time of arrival of the photons associated to a secondary muon track is used to reconstruct the neutrino direction and energy. This basic principle is adopted for the KM3NeT neutrino telescope [1]; the target material will be the water in the deep Mediterranean sea. This telescope is composed of a number of vertical structures (the "Detection Units": DUs), which are anchored to the sea bed and kept vertical by one or several buoys at their tops. Each DU carries photo-sensors and further devices for calibration. The basic photo-sensor unit is an "Optical Module (OM)" housing with several Photo Multiplier Tubes (PMT's), their high-voltage bases and their interfaces to a nanosecond precision data acquisition system. The KM3NeT research infrastructure [2] will be designed to survive at least a decade in the deep sea, under high pressure and in an aggressive salt water environment.

The OM is a densely packed glass envelope. Keeping the dissipation of the electronics within safe limits demands severe power budgeting. The cooling can handle about $10 \mathrm{~W}$ for the complete functionality. To fulfill these requirements the PMT circuit has to be designed for a very low dissipation.

One of the major demands for the PMT electronics is providing a stable high voltage for all the dynodes. The described system uses a Cockcroft Walton multiplier for the high voltage stack driven by a flyback converter. State of the art, commercially available circuits dissipate in and around $50 \mathrm{~mW}$. This circuit has been optimized to dissipate very low power. 


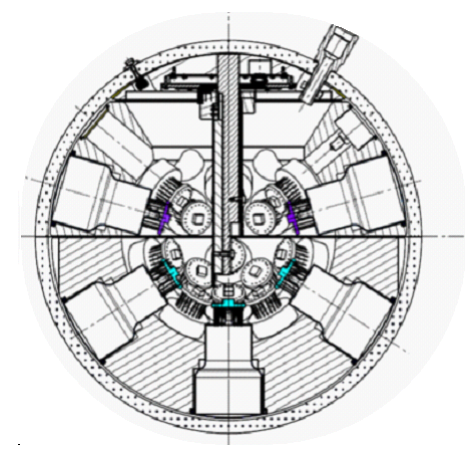

Figure 1. Possible design of a multi PMT optical module.

\begin{tabular}{|l|l|c|}
\hline Supply voltage & $800<1400$ & V \\
\hline Gain & $5 \times 10^{6}$ & \\
\hline Gain slope (vs supp. Volt., log/log) & 6.8 & \\
\hline Voltage cathode-D1 & $150<500$ & $\mathrm{~V}$ \\
\hline Consecutive dynode & $<300$ & $\mathrm{~V}$ \\
\hline Anode and D10 & $40<300$ & $\mathrm{~V}$ \\
\hline
\end{tabular}

Table 1. Main parameters of the PMT-base.

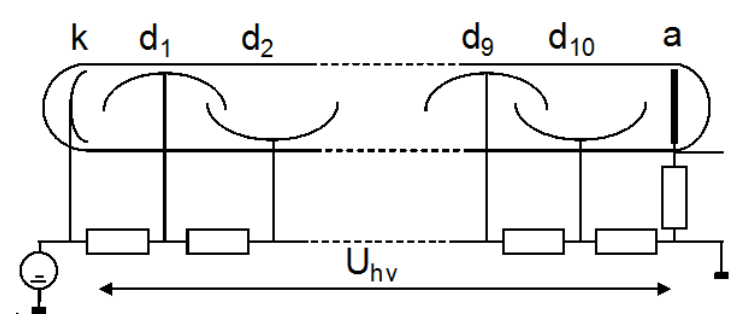

Figure 2. Resistive High-voltage divider.

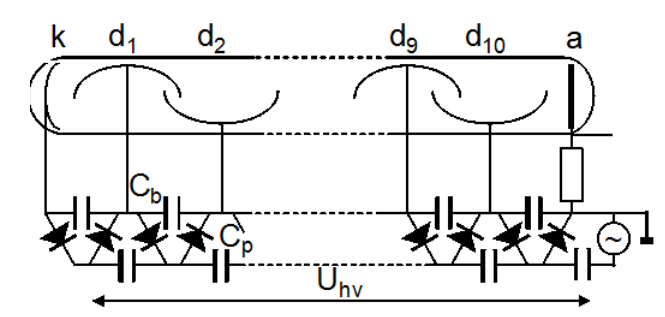

Figure 3. Schematic of the Cockcroft Walter Multiplier.

\section{System components}

\subsection{Photo Multiplier Tube}

Figure 1 shows a glass sphere, also called OM (size: 17") which will house thirty one PMTs (each of size: 3"). The main electrical parameters of the PMT are listed in table 1 [2].

The PMT has 10 dynodes and is used with the following voltage ratio scheme;

$\mathrm{K}: \mathrm{DA}: \mathrm{G}: \mathrm{D} 1: \mathrm{D} 2: \mathrm{D} 3: \mathrm{D} 4: \mathrm{D} 5: \mathrm{D} 6: \mathrm{D} 7: \mathrm{D} 8: \mathrm{D} 9: \mathrm{D} 10: \mathrm{A}$

$0.15: 1: 1.5: 2: 1: 1: 1: 1: 1: 1: 1: 1: 1$

One option to supply all the PMT's with the high voltage would be to design a common high voltage supply. Disadvantages to this option are:

1. the differences between the tubes and the need for resistive voltage dividers for each tube to prevent cross talk (figure 2) [3]

2. Risk of a single point of failure.

3. Resistive dividers dissipate a lot of power. 


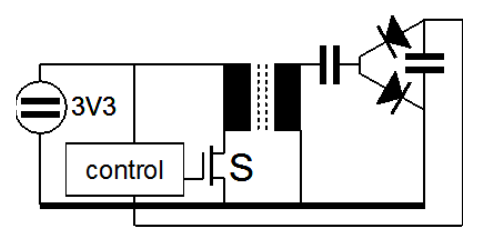

Figure 4. A flyback converter with first stage of a CW.

Matching of the performance of each tube is achieved by an adjustable resistive divider for each tube.

\subsection{Cockcroft Walton multiplier (CW)}

A very interesting and efficient alternative is the Cockcroft Walton multiplier or the Villard cascade (figure 3). In this case energy from an alternating source is transferred by pumping capacitors through rectifiers into buffer capacitors near the dynodes [4]. In single photon count mode, the electron current in the tube will be very low and the buffer capacitor holds only the charge to adjust the dividers on the right level.

The circuit is ideal for this application (neutrino telescope). The PMT requires the highest current at the last dynode, next to the anode. This is attached to the lowest stage of the CW chain (the lowest voltage), the point at which the chain can supply the highest current. At the higher stages of the $\mathrm{CW}$ chain (the highest voltage), where it is much harder for the $\mathrm{CW}$ multiplier to supply current, the PMT requires only a few electrons for each hit. Fine tuning of the voltage levels near the cathode is done by high value resistors as shown in the PMT base schematic and they will hardly dissipate. So the high-voltage generation is very efficient.

\subsection{Flyback converter}

Flyback converters are often used in electronic equipment for low power consumption. Because of the low number of components compared to other converters [5]. In this design, both the forward and the flyback phase are used (figure 4).

In the on-state of switch S, energy is transferred from the input voltage source by the transformer and pump capacitor into the load capacitor. In the off-state, the energy, stored in the transformer, is used to reset the pump capacitor (flyback). Radio Frequency interference is a critical issue and necessitates a careful design of the PCB and careful selection of components for such a converter.

\section{Design}

Figure 5 shows the complete circuit design. The flyback control circuit, ADP1111 [6], contains the power switch $\mathrm{S}$ of figure 4 and drives the primary of the flyback transformer (L1a). Inductor L3 limits the current rise time during the first part of the on-state of switch S. By choosing a low maximum pulse frequency, requirements such as: power, electromagnetic compatibility (EMC) and controlled rise and fall times, can be achieved. The ADP1111 has a maximum on time of 8 $\mu \mathrm{s}$. In this time energy is stored in the transformer. The ADP1111 regulates the high voltage with 


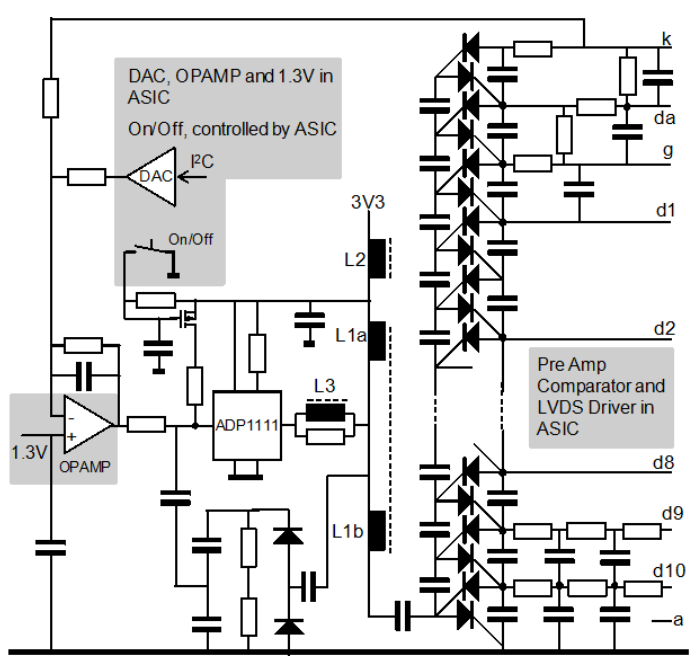

Figure 5. Block diagram of the system.

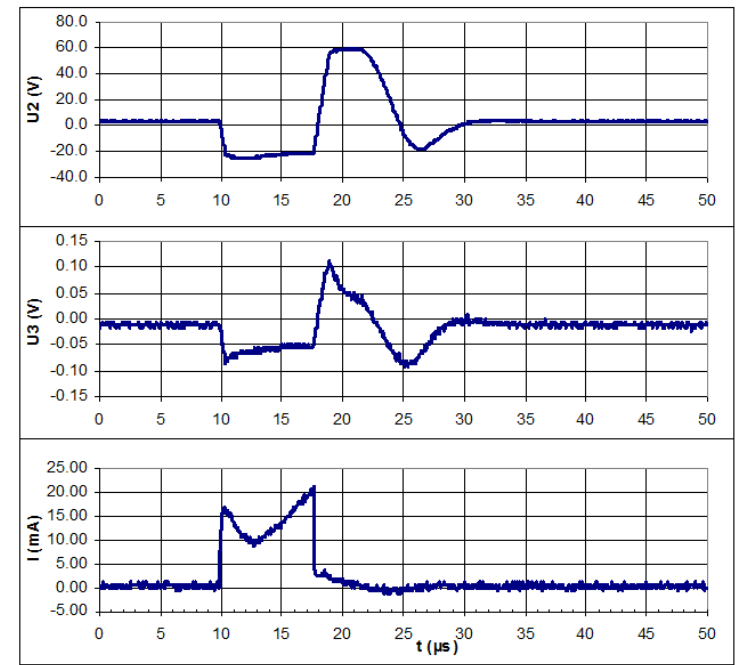

Figure 6. Upper trace: HV output of L1, middle trace: AC signal of dynode 10, bottom trace: load current of L1.

the pulse repetition rate, driven by a positive feedback, up to $100 \mathrm{~V}$ to the $\mathrm{CW}$ chain. An overdrive on the control input of the ADP1111 together with the output signal of the DC feedback op-amp forces single period burst. In the top of the $\mathrm{CW}$, a circuit is placed to adjust the voltages of the electron focusing part of the PMT. This circuit provides filtering to the most sensitive part of the PMT for so called 'dark-count' pulse generation and transit time variations. Component values are chosen in a way to prevent reverse polarities during start up. Inductor L2 is used to decouple the circuit from its neighbors and the ASIC placed on the other side of the printed circuit board.In the dynode circuit of $\mathrm{d} 9$ and $\mathrm{d} 10$ extra resistors and capacitors are placed to filter the dynode ripple and to limit the anode current in case of an over exposure of the PMT. Design of the printed circuit board (PCB) is done in a way to adapt HV components (leak, corona) and EMC rules. The PCB was designed very carefully and forms an integral part of the system requirements and must not be viewed as just a carrier of components. To achieve a good reliability special attention is paid to cooling issues in the OM to keep temperature as low as possible.

\section{Results}

\subsection{Waveforms}

All the measurements shown in figure 6 are made with $3.3 \mathrm{~V}$ supply voltage and $1050 \mathrm{~V}$ cathode voltage. The waveforms are consistent with the simulations. The upper trace shows the secondary voltage behavior of a complete conversion cycle. The first negative part is the forward period, the positive second part is the flyback period and the following negative part shows the damping of the ringing of the transformer. The middle trace shows the ripple voltage on a dynode and lower trace shows the current in the transformer primary (L1a). The first triangle is forward current due to the charging of the buffer capacitors and leakage inductance of the transformer. The second triangle is because of the magnetization current of the transformer. Both effects are balanced by 


\begin{tabular}{|l|l|}
\hline Ripple dynode to gnd & $150 \mathrm{nV}_{\mathrm{pp}} \mathrm{dV} / \mathrm{dt}<75 \mathrm{mV} / \mathrm{\mu s}$ \\
\hline Inter dynode ripple & $<<200 \mathrm{mV} V_{p p}$ \\
\hline Repetition rate & See figure 7 \\
\hline Voltage input & $2.5-4$ V, Load $<4.5 \mathrm{~m}$ W \\
\hline Voltage output & $800-1200 \mathrm{~V}$ \\
\hline Stab. factor & $0.95 \%$ output at $38 \%$ input \\
\hline Settling Time & $6 \mathrm{~s}$ \\
\hline Power during switch on & $<50 \mathrm{~mm}$ \\
\hline RFI radiation 150 $\mathrm{kHz}-2 \mathrm{MHz}$ & $-20 \mathrm{~dB}$ \\
\hline
\end{tabular}

Figure 7. Global results of this design.

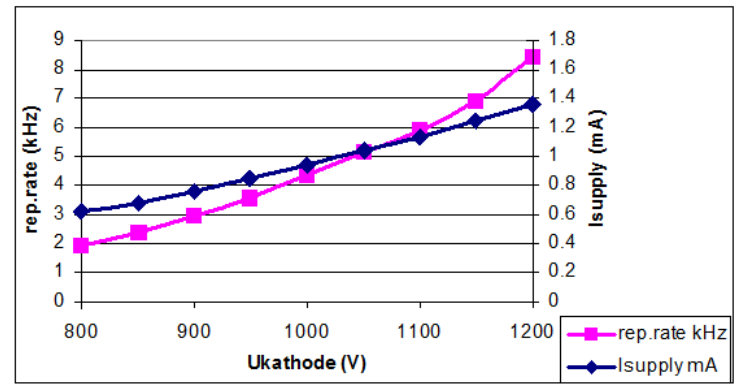

Figure 8. Load and rep. rate for different output levels.

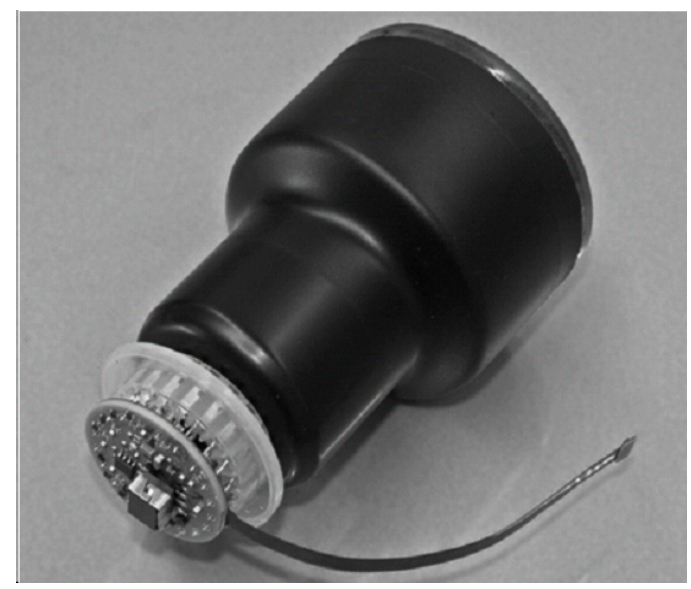

Figure 9. Working prototype $(38 \mathrm{~mm} \varnothing)$.

optimizing the components (L3//resistor) to prevent high di/dt's during switching on and off. See table 7 for more specifications. Measurements show no influence of the achieved ripple on the PMT signal current.

The influence of the cathode voltage level on power supply current and the switching frequency of ADP1111 is shown in figure 8 . The power dissipation will therefore vary, at $3.3 \mathrm{~V}$, between 2 and $4.5 \mathrm{~mW}$.

\section{Outlook}

With this design, shown in figure 9, the total power dissipated for HV generation in an optical module is about $31 \times 3.3 \mathrm{~mW}=102.3 \mathrm{~mW}$ compared to $31 \times 50 \mathrm{~mW}=1.55 \mathrm{~W}$ by commercially available state of the art PMT power supplies. All the switching components, like diodes and pump capacitors, are placed on the off PMT side of the PCB. On the PMT side, the buffer capacitors, the analog signal path: preamplifier, comparator and LVDS driver are placed. In the final design, the analog signal path is integrated in a full custom ASIC ( $350 \mathrm{~nm}$ technology) along with two DAC's for HV tuning and threshold setting. Wider applications of this design could be in apparatus demanding HV sources like handheld (and other) radiation measurement equipment, medical imaging equipment, medical scan equipments etc. 


\section{Acknowledgments}

This work is part of the research program of the 'Stichting voor Fundamenteel Onderzoek der Materie (FOM)', which is financially supported by the 'Nederlandse Organisatie voor Wetenschappelijk Onderzoek (NWO)'.

\section{References}

[1] http://www.km3net.org, http://www.km3net.org/.

[2] http://www.km3net.org/CDR/CDR-KM3NeT.pdf.

[3] S.O. Flyckt et al., PMT principles and applications, Photonis (2002); http://www.photonis.com/.

[4] Cockcroft-Walton Voltage multipliers, http://www.techlib.com/files/voltmult.pdf.

[5] M. Brown, Power Supply Cookbook, Butterworth-Heinemann (2001).

[6] Datasheet of ADP1111 from Analog Design, http://www.analog.com/en/index.html. 